\title{
REGISTRO DE TEMPERATURA USANDO \\ EL MICROCONTROLADOR PIC16C71
}

\author{
José González Flórez ${ }^{*}$ Eusebio García García
}

\begin{abstract}
A digital measurement temperature employs the PIC $16 C 71$ microcontroller, which has been used as an analog to digital converter. The LM 335 is used as the temperature sensor which is linear in the range $-40^{\circ} \mathrm{C}$ to $120^{\circ} \mathrm{C}$ with a variation of $1 \mathrm{mV} / \mathrm{C}$. The data is shown using leds.

The data is captured programming the JW version of the Microchip PIC $16 C 71$ following the instruction of the "PIC 16/71 MICROCONTROLLER DATA BOCK".
\end{abstract}

\section{Resumen}

En este trabajo se presenta el registro de temperatura de una manera digital, empleando el microcontrolador PIC 16C71 configurado como conversar análogo-digital. El sensor de temperatura es el integrado LM335 que es lineal en el intervalo de -40 a 120 oc, con variación de $1 \mathrm{mV} /{ }^{\circ} \mathrm{C}$. El dato correspondiente se muestra en diodos leds.

La lectura se realiza configurando por programa el PIC 16C7 de Microchip en versión JW, de acuerdo a las indicaciones del manual de referencia "PIC 16/17 MICROCONTROLLER DATA BOCK".

\section{DESCRIPCIÓN DEL MICROCONTROLADOR PIC 16C71}

\subsection{Características}

EI PIC 16C71 es un microcontrolador de rango medio, fabricado por MICROCHIP, el cual posee las siguientes características:

a. 18 pines, 13 son entrada/salida, dirección individual por programa (Figura 1).

b. Velocidad de operación desde DC a $16 \mathrm{Mhz}$.

c. Memoria de programa EPROM de 1024 posiciones.

d. Cuatro canales $A / D$, de 8 bits y resolución \pm 1 bit.

e. 36 registros de propósito general y 15 registros especiales

f. Pila de 8 niveles.

g. Cuatro fuentes de interrupción.

h. Los pines pueden manejar leds de manera directa.

i. Temporizador/contador de 8 bits, con preescalador.

j. Circuito de vigilancia.

k. Cuatro opciones de oscilador.

I. Seguridad en protección al programa.

\footnotetext{
*Profesor Asociado. Universidad Pedagógica Nacional.

** Profesor Catedrático. Universidad Pedagógica Nacional.
} 
m. Modo de bajo consumo

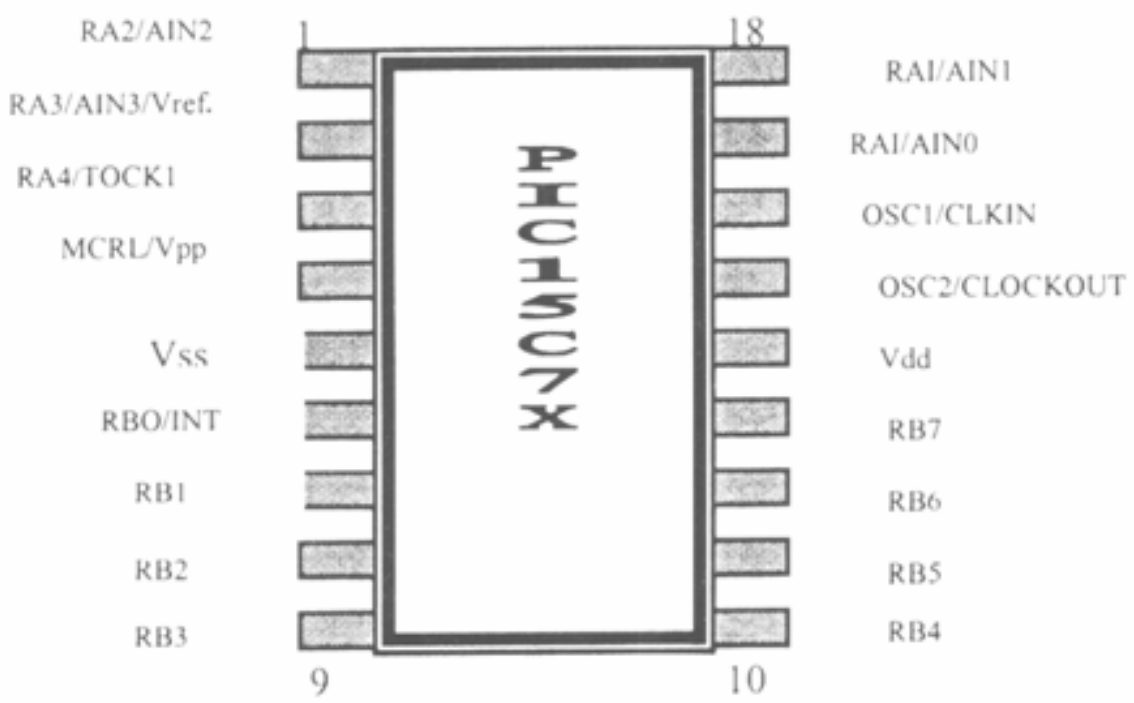

Figura 1. Distribución de pines en el integrado visto desde arriba.

\subsection{Distribución de la memoria de programa en el pic}

El registro PC los cuales son PCL (bajo) y PCLATH (alto) indica la línea de programa que está ejecutando, la cual se puede leer o escribir para ubicarse en el programa (figuras 2 y 3$)$.

El programa se inicia en la línea 0000h, y retorna a esta en el reset. Cuando se presenta interrupción el programa ejecuta la línea 0004h (figura 2).

Figura 2.

Mapa de la memoria de programa y pila.

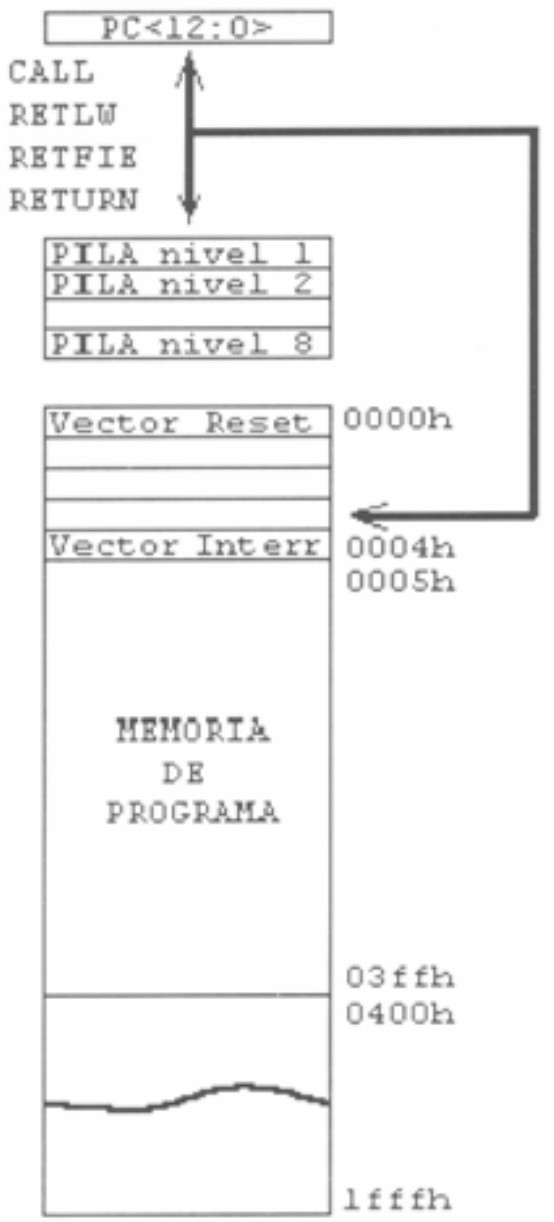




\subsection{Posición de los registros}

Los registros se clasifican en especiales y de propósito general. Los primeros están ubicados en la dirección 00 a $0 \mathrm{~B}$ y de 80 a 8B, los cuales configuran la operación del microcontrolador cada vez que el programa realiza una operación sobre ellos. Los registros generales solo almacenan datos, mientras el microcontrolador está en operación y se ubican en las posiciones $0 \mathrm{C}$ a $2 \mathrm{~F}$.

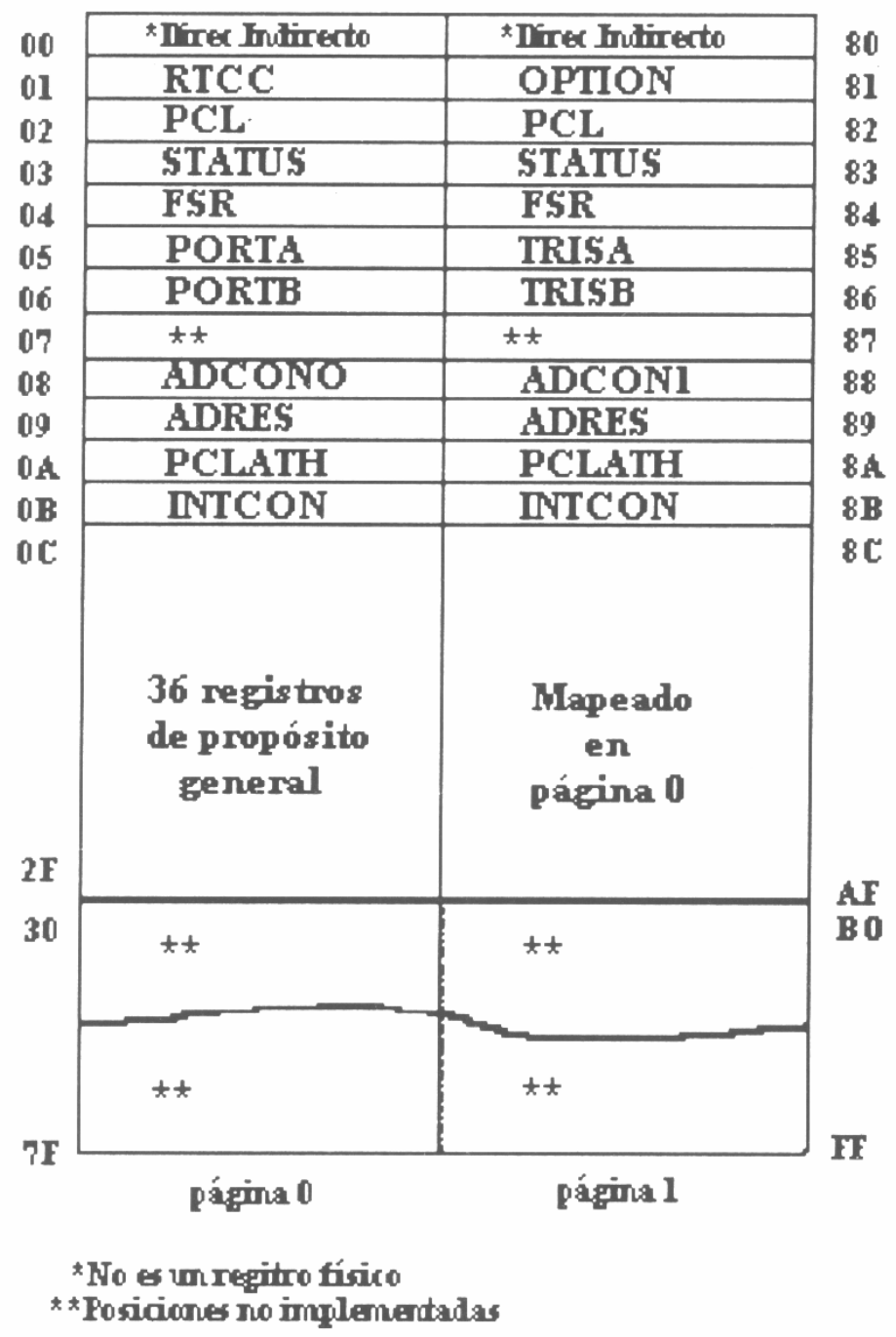

Figura 3. Organización de registros.

\subsection{Instrucciones del microcontrolador Operaciones orientadas a registros}

Mnemotécnico

$\begin{array}{ll}\text { ADDWF } & \mathrm{f}, \mathrm{d} \\ \text { ANDWF } & \mathrm{f}, \mathrm{d} \\ \text { CLRF } & \mathrm{f} \\ \text { CLRW } & - \\ \text { COMF } & \mathrm{f}, \mathrm{d} \\ \text { DECF } & \mathrm{f}, \mathrm{d} \\ \text { DECFSZ } & \mathrm{f}, \mathrm{d} \\ \text { INCF } & \mathrm{f}, \mathrm{d}\end{array}$

Operación

Suma W y $f$

AND entre $W$ y $f$

Limpiar $f$

Limpiar W

Complementar $f$

Decrementar $f$

Decrementar $\mathrm{f}$, saltar si cero

Incrementar $f$
Estados afectados

$\mathrm{C}, \mathrm{DC}, \mathrm{Z}$

Z

Z

Z

Z

Z

Z 


\begin{tabular}{|c|c|c|c|}
\hline INCFSZ & $f, d$ & Incrementar f, saltar si cero & \\
\hline IORWF & $f, d$ & Or entre $\mathrm{W}$ y $\mathrm{f}$ & Z \\
\hline Mnemoté & & Estados afe & os \\
\hline MOVF & f., $d$ & Mover $\mathrm{f}$ & Z \\
\hline MOVWF & $f$ & Mover W a f & \\
\hline NOP & - & No operación & \\
\hline RLF & $f, d$ & Rotar a la izquierda a través de carry & C \\
\hline RRF & $f, d$ & Rotar a la derecha a través de carry & C \\
\hline SUBWF & $f, d$ & Restar W de f & C, DC, Z \\
\hline SWAPF & $f, d$ & Intercambiar nibbles de f & \\
\hline XORWF & $\mathrm{f}, \mathrm{d}$ & Or exclusiva entre $\mathrm{W}, \mathrm{f}$ & Z \\
\hline
\end{tabular}

\section{Operaciones orientadas a bits}

$\begin{array}{lll}\text { BCF } & f, b & \text { Limpiar bit b de } f \\ \text { BSF } & f, b & \text { Activar bit b de } f \\ \text { BTFSC } & f, b & \text { Probar bit b de } f \text {, saltar si es cero } \\ \text { BTFSS } & f, b & \text { Probar bit b de f, saltar si es uno. }\end{array}$

\section{Operaciones literales y de control}

\begin{tabular}{|c|c|c|c|}
\hline ADDLW & $\mathrm{k}$ & Sumar literal k a W & C, DC, Z \\
\hline ANDLW & $\mathrm{k}$ & AND entrekyW & Z \\
\hline CALL & $\mathrm{k}$ & Llamar subrutina & \\
\hline CLRWDT & - & Limpiar WDT & /T0, /PD \\
\hline GOTO & $\mathrm{k}$ & Saltar a la dirección $\mathrm{k}$ & \\
\hline IORLW & $\mathrm{k}$ & Or entre k y W & Z \\
\hline MOVLW & $\mathrm{k}$ & Cargar a W con literal $\mathrm{k}$ & \\
\hline RETFIE & - & Retornar de interrupción & \\
\hline RETLW & $\mathrm{k}$ & Retornar y cargar a W con $\mathrm{k}$ & \\
\hline RETURN & - & Retornar de subrutina & \\
\hline SLEEP & - & Ir al modo de bajo consumo & /T0, /PD \\
\hline SUBLW & $\mathrm{k}$ & Restarle k a W & $\mathrm{C}, \mathrm{DC}, \mathrm{Z}$ \\
\hline XORLW & $\mathrm{k}$ & Or exclusiva entre $\mathrm{k}$ y $\mathrm{W}$ & Z \\
\hline
\end{tabular}

\section{DISEÑO DEL SENSOR}

El montaje del sensor se muestra en la figura 4, en forma esquemática, y su respectivo circuito impreso en la figura 5. El análisis es como sigue: el voltaje que es dependiente de la temperatura dado por el integrado LM335 se conecta en el Pín 3 del potenciómetro P1, la variación del potenciómetro P1 da la sensibilidad de la escala.

El voltaje que proviene del pín 7 del amplificador operacional U1 B es sumado a la del pín 3 del potenciómetro $\mathrm{P} 1$ ajustando el potenciómetro $\mathrm{P} 2$, de tal manera que asegura el valor de referencia o mínimo valor de la escala. En el pín 8 del amplificador operacional al variar el potenciómetro P3 se obtiene un multiplicador inversor, y finalmente el amplificador operacional U1D invierte la tensión y es la señal que se introduce al microcontrolador. 
Es de anotar que la escala debe ser tal que la referencia se debe colocar en $0 \mathrm{~V}$ y su máximo valor en $5 \mathrm{~V}$, por ello se deben colocar diodos Zener de protección.

Materiales:

13 resistencias de $10 \mathrm{~K}$ Ohmios a 1/4 Watio, 3 potenciómetros lineales de 10K Ohms, amplificador operacional LF 347, 2 conectores machos de 4 y 2 pines.

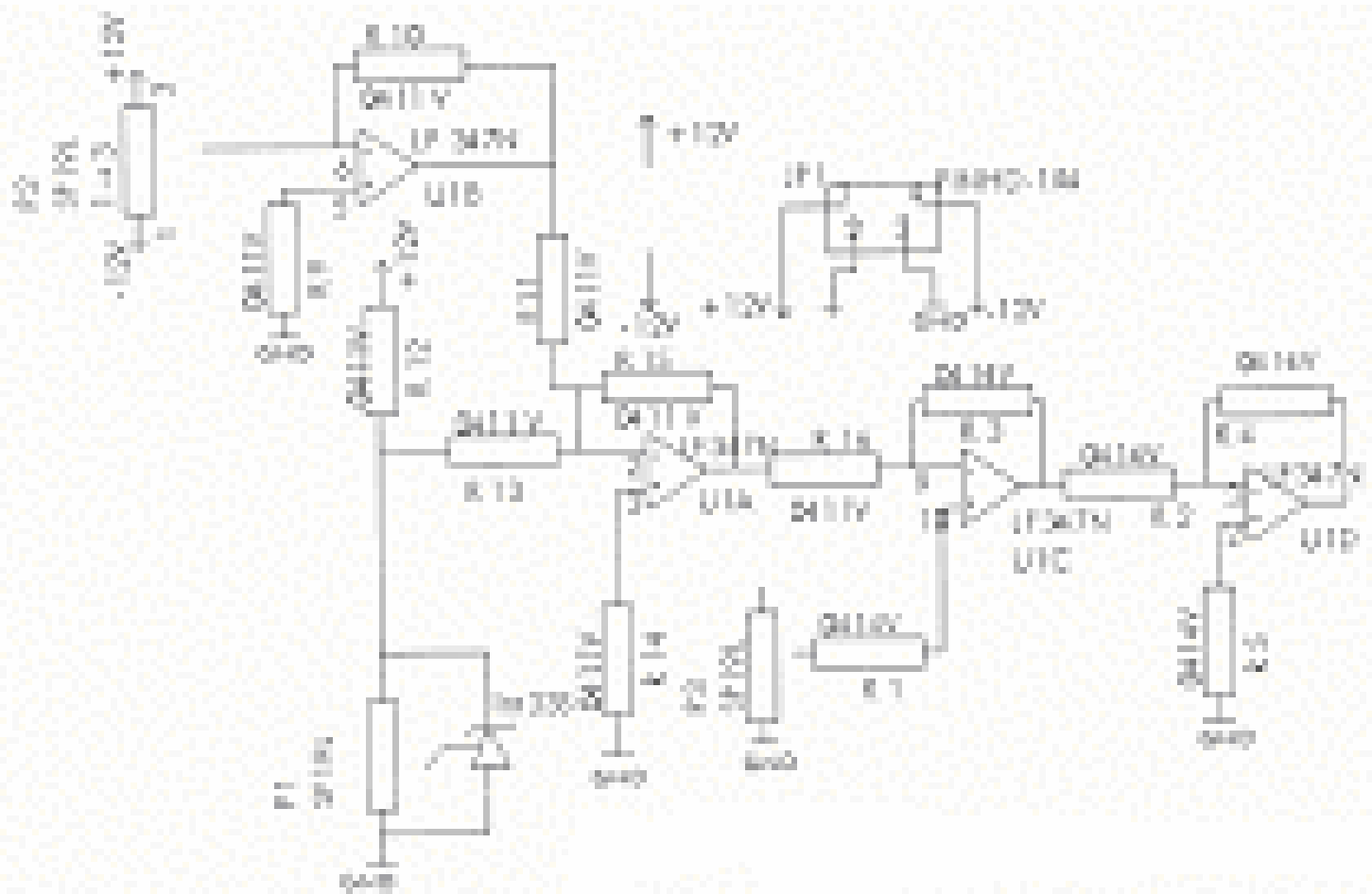

Figura 4. Diagrama del sensor de temperatura.

El circuito impreso es de una sola faz, la cual minimiza los costos y facilita la manipulación del sensor.

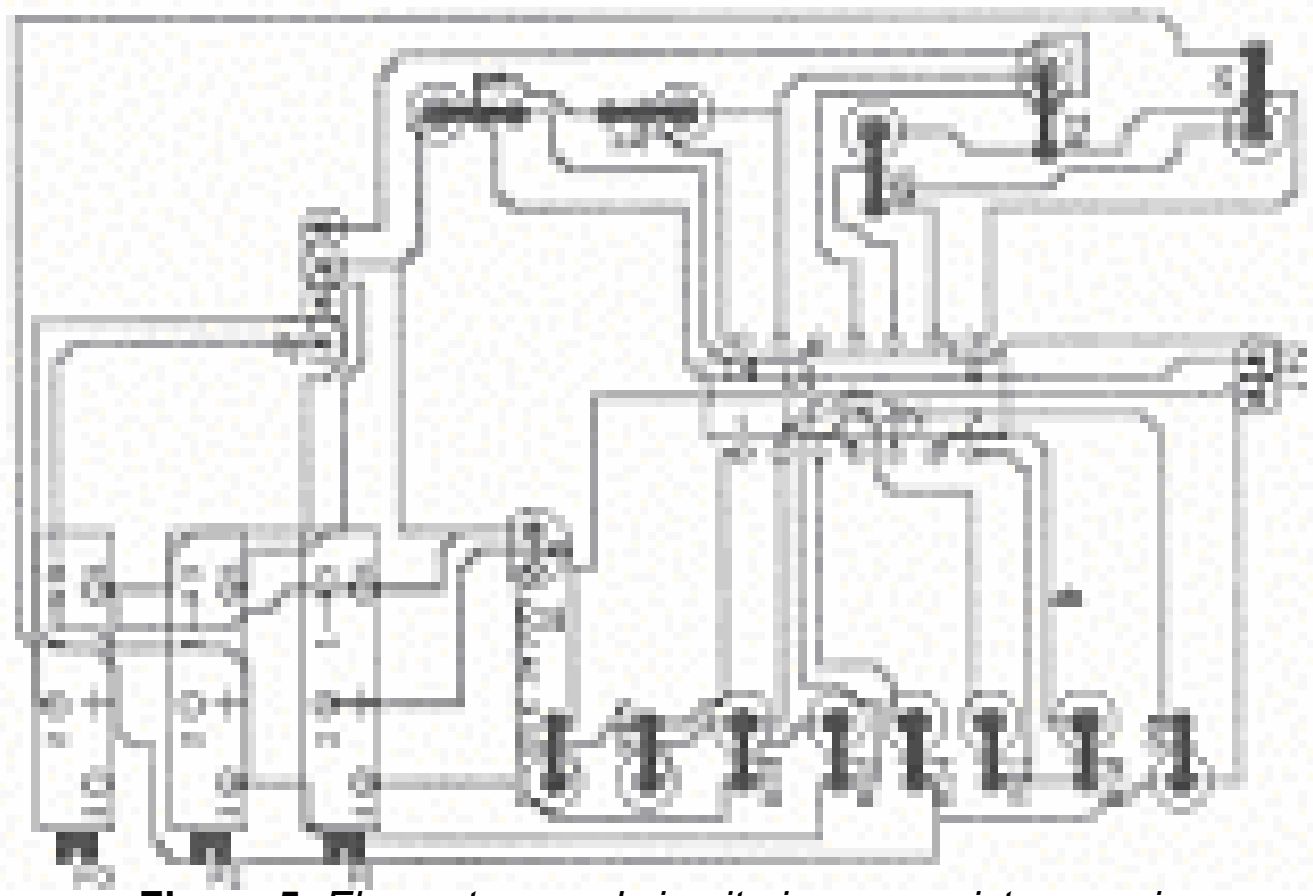

Figura 5. Elementos en el circuito impreso, vista superior. 


\section{FUNCIONES ASIGNADAS POR PROGRAMA AL MICROCONTROLADOR}

- La conversión AID, con canal previo seleccionado.

- Manejo de tiempos base de $1 \mathrm{~ms}$.

El funcionamiento del microcontrolador depende del programa consignado en la memoria de programa" y de los valores que presenten los registros especiales y generales en la "memoria de registros".

La utilización del microcontrolador ahorra instrumentación simplificando la tarjeta de la interface, su rapidez máxima de 4 millones de instrucciones por segundo hace que para tiempo real pueda realizar operaciones matemáticas (sumas, restas, multiplicaciones, divisiones, derivadas, integrales, la transformada discreta de Fourier, etc.). Además, toma decisiones para hacer control sobre los elementos (activación de relés, fototransistores, triac, etc.) o variables que está registrando (luz, temperatura, presión, voltaje, corriente, resistencia, etc.), dependiendo de la configuración de la interface

Las posibilidades que ofrece el microcontrolador para resolver un problema son ilimitadas, solo la experiencia y conveniencia del programador decide la rutina a utilizar.

\subsection{Conversión A/D}

La conversión A/D se configura dentro del programa principal como una subrutina y sus pasos son:

a) Configurar el módulo A/D:

- Definir pines análogos.

- Referencia de voltaje.

- Entradas y salidas digitales.

- Selección del canal A/D de entrada.

- Selección del reloj de conversión.

- Habilitar el módulo de conversión.

- Si requiere interrupción:

- Colocar en cero el bit ADIF.

- En uno a los bits ADIE y GIE.

b) Espera de tiempo mínimo dado por el reloj utilizado.

c) Inicio de conversión, colocando en uno a GO/DONE (ADCONO).

d) Espera para terminar la conversión, las banderas de señales son: Cero para GO/DONE y uno para ADIF.

e) Leer el resultado de conversión en ADRES, colocar cero en ADIF si es requerido.

f) Si se desea otra conversión en el mismo canal se traslada al punto b), con nuevo canal al punto a).

Configuración utilizada:

PIC utilizado: P16C71.

Cuatro canales análogos.

Conversión por el canal ANO.

Voltaje de referencia RA3.

Cristal de 16 Mhz.

No utiliza la interrupción. 
Subrutina que realiza una sola conversión:

\begin{tabular}{|c|c|c|}
\hline \multirow[t]{8}{*}{ Conv_A/D } & bsf & STATUS, RPO \\
\hline & movlw & b00000001; tres canales análogos \\
\hline & movwf & ADCON0; Voltaje de referencia: RA3 \\
\hline & & STATUS, RPO \\
\hline & movlw & $\begin{array}{l}\text { b'10000000; Fosc/32, canal AN0, } \\
\text { ADCON0. deshabilita AID }\end{array}$ \\
\hline & & ADCONO, ADON; habilitar modulo A/D. \\
\hline & Movlw. & 189; $(256-67)^{*} 3^{*} 250 * 10^{-9} \mathrm{~s}=50$ us. \\
\hline & movwf & DEMORA \\
\hline \multirow[t]{3}{*}{ DEM } & incfsz & DEMORA, W \\
\hline & goto & DEM \\
\hline & bsf & ADCON0, GO; Inicio de la conversión \\
\hline \multirow[t]{10}{*}{ CGO } & btfsc & ADCON0, GO; Preguntar por el fin \\
\hline & goto & CGO \\
\hline & movf & ADRES, W; guardar el resultado en \\
\hline & movwf & CONVERSION; el registro CONVERSION \\
\hline & & STATUS, RP0; retornar los pines \\
\hline & movlw & b'11111111'; análogos a digitales \\
\hline & movwf & ADCON1 \\
\hline & & STATUS, RP0 \\
\hline & movf & CONVERSION, W; mueve f \\
\hline & movwf & PORTB; puerto B \\
\hline
\end{tabular}

\subsection{Manejo de tiempo}

Para dar un lapso de espera entre conversiones se requiere un intervalo de tiempo que se ejecuta como sigue: para definir intervalos, producir o contabilizar eventos en un lapso de tiempo se realiza por el registro TMRO o definiendo sus incrementos por ciclos internos del oscilador según la configuración del preescaler establecido. Cuando hay desbordamiento de TMR0, se presenta una bandera señalando que el evento ha ocurrido y puede incrementar otro registro, además, se puede programar interrupción para considerarlo como evento especial.

\section{LIMITACIONES DE LA INTERFACE}

Los elementos utilizados tienen garantías dadas por el fabricante, para asegurar un buen desempeño en el trabajo se requiere observar atentamente sus características: máximo tiempo de operación, tensiones de entrada y salida, y tensiones de alimentación (ver bibliografía 3).

El microcontrolador puede realizar conversiones cada $50 \mathrm{~ms}$.

Para proyectos que requieren mayor velocidad de conversión A/D es necesario acudir a los conversores de comunicación y estos exigen el almacenamiento de datos en memorias volátiles. 


\section{CONCLUSIONES}

Como se puede apreciar en este trabajo se hace la presentación del microcontrolador PIC16C71 (tecnología de punta) en solo una de sus múltiples funciones, la conversión análoga digital $A / D$. El empleo de los microcontroladores abre la posibilidad de interactuar con diversos circuitos integrados que permiten recibir y enviar datos procesados por el mismo a través de las rutinas del programa. Esto quiere decir que se puede obtener y dar información al mundo físico de una manera casi ilimitada. Se puede conectar a diversas interfaces y particularmente con las PC de una forma inmediata.

Con esto surgen otras posibilidades para que los estudiantes se vinculen a realizar sus trabajos utilizando el PC corno aparato de registro, a su vez permiten que las instituciones puedan dar un uso adecuado del equipo de cómputo que se encuentra obsoleto y almacenado.

El empleo como interface de cualquier microcontrolador convierte a los PC en elementos valiosos en el laboratorio con la gran ventaja que su conexión se hace a través de los puertos de la impresora o del Mouse, sin recurrir a destapar el equipo.

\section{BIBLIOGRAFÍA}

1. EleCtRÓNICA Y COMPUTADORES, 1994, ed. Cekit. Pereira. Año 1. Nº 8.

2. EMBEDDED CONTROL HANDBOOK.1997, ed, Microchip. USA.

3. PIC 16/17 MICROCONTROLLER DATA BOOK.1997, ed. Microchip. USA. 